Check for updates

Cite this: RSC Adv., 2018, 8, 15522

\title{
Enhancing a clenbuterol immunosensor based on poly(3,4-ethylenedioxythiophene)/multi-walled carbon nanotube performance using response surface methodology $\dagger$
}

\author{
Nurul Ain A. Talib, ${ }^{\text {ab }}$ Faridah Salam, ${ }^{c}$ Nor Azah Yusof, ${ }^{\text {ab }}$ Shahrul Ainliah Alang \\ Ahmad, (D) ${ }^{\text {ab }}$ Mohd Zulkhairi Azid, ${ }^{d}$ Razali Mirad ${ }^{d}$ and Yusran Sulaiman (D) *ab
}

Clenbuterol (CLB) is an illegal antibiotic for livestock, which is misused as a growth promoter drug. In this study, an immunosensor modified with poly(3,4-ethylenedioxythiophene) (PEDOT), multi-walled carbon nanotubes (MWCNT) and anti-clenbuterol antibody (Ab) was developed for the detection of CLB. A screen-printed carbon electrode (SPCE) was modified with PEDOT/MWCNT as a sensor platform before immobilizing $A b$ for specific CLB binding through a competitive-type immunoassay. Free CLB in the sample solution competed with clenbuterol-horseradish peroxide (CLB-HRP) to bind with Ab. A high current signal was obtained after optimization of the electrochemical immunoassay conditions $(\mathrm{pH}$, incubation temperature, antigen (Ag) incubation time and \% blocking) using the response surface methodology/central composite design (RSM/CCD). The developed immunosensor is highly reproducible and sensitive with good storage stability, which are necessary for practical application. In real sample application, this immunosensor produces comparable results with liquid chromatographymass spectrometry; thus, it is useful for CLB screening and monitoring in real meat samples.

Received 5th January 2018

Accepted 8th April 2018

DOI: 10.1039/c8ra00109j

rsc.li/rsc-advances fever and respiratory problems in human, which are influenced by CLB residue in meat products. ${ }^{4}$ Therefore, the use of this antibiotic in meat products requires continuous monitoring to avoid pharmacological effects to consumers.

In previous studies, a few methods were developed to detect and monitor antibiotics including CLB such as microbiology assay, enzyme-linked immunosorbent assay (ELISA), ${ }^{5}$ high performance liquid chromatography (HPLC), ${ }^{6,7}$ liquid chromatography-mass spectrometry (LC-MS), ${ }^{8}$ gas chromatography-mass spectrometry (GC-MS), ${ }^{9}$ capillary electrophoresis, ${ }^{10,11}$ surface plasma resonance ${ }^{12}$ and electrochemical ${ }^{13}$ methods. However, even though analytical methods such as HPLC, LC-MS and GS-MS are very sensitive and effective, these methods are relatively expensive, time-consuming and unsuitable for on-site monitoring due to the use of large instruments.

Electrochemical sensors can be developed into various intradisciplinary applications such as food monitoring, ${ }^{14}$ pharmaceutical ${ }^{15}$ and pesticide detection, ${ }^{16,17}$ wastewater screening, ${ }^{18,19}$ chemical sensors ${ }^{20}$ and biosensors ${ }^{21}$ based on the modification of the sensor platform. Many methods have been developed by utilizing various materials such as molecular imprinted polymers, ${ }^{22,23}$ antibodies (Ab) ${ }^{24,25}$ and enzymes ${ }^{26,27}$ to increase the sensitivity, selectivity and effectiveness of electrochemical detection. 
A biosensor that utilizes $\mathrm{Ab}$ to capture antigen $(\mathrm{Ag})$ is known as an immunosensor. ${ }^{28}$ Immunosensors based on modified electrode surfaces are fabricated using various materials to enhance their electrochemical properties, which improves the electrode charge transfer, produces a better signal and increases the sensitivity. Carbon nanotubes (CNTs) have good conductivity $^{29}$ and provide many binding sites for $\mathrm{Ab}$ binding due to their high surface area, and thus are suitable for the development immunosensors. ${ }^{30,31}$ The electrochemical properties of CNT can be enhanced by incorporating conducting polymers such as polyaniline, ${ }^{32}$ polypyrrole, ${ }^{33}$ and poly(3,4ethylenedioxythiophene) (PEDOT). ${ }^{34}$ Their biocompatibility and ability to improve the efficiency of bio-recognition events have been reported as the advantages of using conducting polymers in the design of immunosensors. ${ }^{35}$ The fabrication of biosensors utilizing PEDOT as one of the materials successfully produced an amperometric glucose sensor. ${ }^{36}$ PEDOT has high stability in phosphate buffer solution ${ }^{37}$ which is very useful for immunoassay and it is also compatible for doping with nanoparticles. A previous study showed that the addition of multiwalled carbon nanotubes (MWCNT) as a dopant into PEDOT improved the electrochemical properties of the composite. ${ }^{38}$

Recently, electrochemical sensors for CLB monitoring have been developed widely since this method is suitable for on-site monitoring. In addition, the detection process can be performed by semi-skilled personnel due to its relatively simple handling process, and it is relatively less expensive ${ }^{39}$ in comparison with other analytical detection methods. CLB is a hapten, which is a very small molecule that has little effect on electron transfer. Herein, a CLB immunosensor is developed by fabricating PEDOT/MWCNT/Ab on a screen-printed carbon electrode (SPCE). SPCEs are reported to be more practical for on-site monitoring. ${ }^{40}$ Additionally, they are inexpensive, ${ }^{41}$ easily modified and reproducible; hence, they are suitable for mass production. By using SPCE, only small amount of sample and electrolyte are needed for each detection process.

Generally, optimization of the immunoassay can enhance the sensitivity of the sensor. One parameter with the one-time approach is usually applied for this purpose, but this method just describes each parameter individually and is unable to evaluate the interaction between parameters. Therefore, a statistical technique such as the response surface methodology (RSM) can be used instead since it indicates the significance of the studied parameters and developed model for better understanding the optimization process. Currently, limited reports are available on RSM for optimization in biosensors. Mirmoghtadaie and co-workers ${ }^{42}$ reported the successful optimization of a DNA electrochemical sensor using RSM for parameters such as $\mathrm{pH}$, concentration of DNA probe and deposition potential. Optimization of the effective parameters for the fabrication of modified electrodes and the immobilization of DNA probes (immobilization time and concentration of DNA) using RSM has enhanced the sensitivity of DNA detection. ${ }^{43}$ The reliability of RSM as an optimization tool was also reported in other literature. ${ }^{\mathbf{4 4 - 4 6}}$

In this work, an electrochemical sensor is designed by utilizing PEDOT, MWCNT and polyclonal anti-clenbuterol antibody $(\mathrm{Ab})$ to produce a high-performance CLB immunosensor. A screen-printed electrode (SPCE) is modified with PEDOT/MWCNT composites through the electrodeposition technique. $\mathrm{Ab}$ is immobilized on the modified surface by exploiting the carboxyl group on the MWCNT walls through the EDC/NHSS protocol, while the direct competitive immunoassay format is adapted for the detection of CLB. This work also focuses on the optimization of the immunoassay conditions $(\mathrm{pH}$, incubation temperature, $\mathrm{Ag}$ incubation time and \% blocking) using the response surface methodology/central composite design (RSM/CCD) approach for the enhancement of the electrochemical detection of CLB. To the best of our knowledge, the optimization of the immunoassay conditions for PEDOT/MWCNT/SPCE using the RSM/CCD approach has not been previously reported. The immunosensing strategy reported here presents a significant electrochemical performance improvement of the sensor platform due to its modification with PEDOT/MWCNT composites. Also, the application of this immunosensor for CLB detection in real samples is validated with LC-MS.

\section{Experimental}

\subsection{Materials and methods}

2.1.1 Chemicals. 3,4-Ethylenedioxythiopehene (EDOT), multi-walled carbon nanotubes (MWCNT), clenbuterol hydrochloride (CLB), salbutamol, terbutaline hemisulfate salt (terbutaline), nitrofurantoin (nitrofuran), vancomycin hydrochloride (vancomycin), tetracycline, chloramphenicol, streptomycin sulfate salt (streptomycin), mabuterol hydrochloride (mabuterol), ractopamine hydrochloride (ractopamine), disodium hydrogen phosphate $\left(\mathrm{Na}_{2} \mathrm{HPO}_{4}\right)$, sodium dihydrogen phosphate $\left(\mathrm{NaH}_{2} \mathrm{PO}_{4}\right), \quad N$-(3-dimethylaminopropyl)- $N^{\prime}$-ethylcarbodiimide hydrochloride (EDC) and $N$-hydroxysulfosuccinimide (NHSS) were obtained from Sigma Aldrich. Concentrated sulphuric acid $\left(\mathrm{H}_{2} \mathrm{SO}_{4}\right)$ was purchased from Friedemann Schmidt, while concentrated nitric acid $\left(\mathrm{HNO}_{3}\right)$ was obtained from Fischer Scientific. Polyclonal anticlenbuterol antibody (Ab) raised against clenbuterol was kindly received from the Malaysian Agricultural Research and Development Institute (Malaysia).

2.1.2 Buffers and solutions. Phosphate buffer solution (PBS) was prepared by mixing $0.01 \mathrm{M} \mathrm{Na}_{2} \mathrm{HPO}_{4}$ and $0.01 \mathrm{M}$ $\mathrm{NaH}_{2} \mathrm{PO}_{4}$ in deionized water and adjusting to the desired $\mathrm{pH}$ accordingly. Crosslinker was prepared by mixing EDC and NHSS in a $1: 1$ ratio in $0.01 \mathrm{M}$ PBS at $\mathrm{pH}$ 7.4. Clenbuterolhorseradish peroxide (CLB-HRP) was diluted in $0.01 \mathrm{M}$ PBS with a $1: 640$ volume ratio, as recommended by the manufacturer (Fitzgerald). The washing buffer was prepared by mixing 0.05\% TWEEN 20 (Sigma Aldrich) with 0.01 M PBS at pH 7.4. Dry milk (Blotto, non-fat Santa Cruz Biotechnology) was used as a blocking agent by diluting in 0.01 M PBS at pH 7.4.

\subsection{Preparation of clenbuterol immunosensor}

MWCNT were treated with an acid mixture of concentrated sulphuric acid $\left(\mathrm{H}_{2} \mathrm{SO}_{4}\right)$ and concentrated nitric acid $\left(\mathrm{HNO}_{3}\right)$ in 
a $3: 1$ ratio. The treated MWCNT were further washed with deionized water until pH 7 was obtained, followed by drying in an oven at $60{ }^{\circ} \mathrm{C}$. Before surface modification, the SPCE underwent pre-treatment by cyclic voltammetry $(\mathrm{CV})$ at -1.5 to $0 \mathrm{~V}$ for 3 cycles in $1.0 \mathrm{M} \mathrm{H}_{2} \mathrm{SO}_{4}$ solution. The PEDOT/MWCNT modified SPCE was prepared by electropolymerization in $0.01 \mathrm{M}$ EDOT in the presence of $0.1 \mathrm{mg} \mathrm{mL} \mathrm{mWCNT}^{-1}$ MW suspension at $1.31 \mathrm{~V}$ for $99.1 \mathrm{~s}^{38}$ via chronoamperometry (CA). All electrochemical experiments were performed using a potentiostat (Autolab PGSTATM101) at a scan rate of $0.1 \mathrm{~V} \mathrm{~s}^{-1}$.

The fabrication of the CLB immunosensor began with the activation of the carboxyl functional groups in the PEDOT/ MWCNT modified electrode surface by dropping $10 \mu \mathrm{L}$ of crosslinker solution (EDC/NHSS) for $15 \mathrm{~min}$ at ambient temperature followed by removal of the unbound crosslinker with washing buffer. The PEDOT/MWCNT/Ab modified electrode was prepared via the incubation of $10 \mu \mathrm{L}$ of Ab solution on the PEDOT/MWCNT electrode surface for $1 \mathrm{~h}$ at $37^{\circ} \mathrm{C}$ followed by rinsing with washing buffer.

The detection of CLB commenced by performing a blocking step which included incubation with $20 \mu \mathrm{L}$ of dry milk for $30 \mathrm{~min}$ at $37{ }^{\circ} \mathrm{C}$ followed by rinsing with washing buffer. The competitive method was applied by adding $10 \mu \mathrm{L}$ of CLB standard ( 0 to $250 \mathrm{ng} \mathrm{mL}^{-1}$ ) or sample solution and $10 \mu \mathrm{L}$ of CLBHRP solution onto the immunosensor during incubation, followed by rinsing with washing buffer. $50 \mu \mathrm{L}$ of tetramethylbenzidine (TMB) was dropped onto the electrode before the chronoamperogram was recorded using a potentiostat for $300 \mathrm{~s}$. The standard calibration graph was fitted with linear regression and the limit of detection (LOD) was determined.

\subsection{Determination of potential applied}

Different applied potentials in the range of -0.6 to $0.6 \mathrm{~V}$ were used for the detection of various concentrations of standard

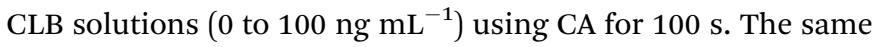
preparation procedure as that for clenbuterol immunosensor was applied here.

\subsection{Optimization of the electrochemical immunoassay condition}

Optimization of the $\mathrm{Ab}$ concentration was conducted by comparing the standard calibration curves at various concentrations of $\mathrm{Ab}\left(10.0,1.0\right.$ and $\left.0.1 \mathrm{mg} \mathrm{mL}^{-1}\right)$. Meanwhile, the other electrochemical immunoassay parameters such as $\mathrm{pH}(A)$, incubation temperature $(B), \mathrm{Ag}$ incubation time $(C)$ and \% blocking $(D)$ were determined by applying RSM/CCD. The study range of each parameter was decided based on previous studies, which were $\mathrm{pH} 6.4$ to $8.4,{ }^{47} 28{ }^{\circ} \mathrm{C}$ to $46{ }^{\circ} \mathrm{C}$ incubation temperature, ${ }^{13} 10$ to $70 \mathrm{~min} \mathrm{Ag}$ incubation time ${ }^{48}$ and 0 to $0.10 \%$ blocking. The experiment was designed based on these ranges and the output of this model was the current response.

A total of 26 experiment runs (Table $\mathrm{S} 1 \dagger$ ) were suggested including 16 factorial points, 8 axial points and 2 center points using the statistical package (Design Expert 6.0, Stat Ease Inc., MN, USA). A mathematical model was generated based on the current response data from the performed experiments to fit the general quadratic model equation (eqn (1)):

$$
Y=\beta_{0}+\sum_{i=1}^{k} \beta_{i} X_{i}+\sum_{i=1}^{k} \beta_{i i} X_{i}^{2}+\sum_{i=1}^{k-1} \sum_{j>i}^{k} \beta_{i j} X_{i} X_{j}
$$

where, $\beta_{0}, \beta_{i}, \beta_{i j}$ and $\beta_{i i}$ represent the intercept, main factor coefficient, two-factor interaction coefficients and quadratic coefficient, respectively. The number of factors $(k)$ is 4 according to the number of variables $\left(X_{1}, X_{2}, X_{3}\right.$ and $\left.X_{4}\right)$ involved. Thus, eqn (1) becomes eqn (2):

$Y=\beta_{0}+\beta_{1} X_{1}+\beta_{2} X_{2}+\beta_{3} X_{3}+\beta_{4} X_{4}+\beta_{11} X_{1}^{2}+\beta_{22} X_{2}^{2}+\beta_{33} X_{3}{ }^{2}+$ $\beta_{44} X_{4}^{2}+\beta_{12} X_{1} X_{2}+\beta_{13} X_{1} X_{3}+\beta_{14} X_{1} X_{4}+\beta_{23} X_{2} X_{3}+\beta_{24} X_{2} X_{4}+$ $\beta_{34} X_{3} X_{4}$

The significance of the developed model was evaluated based on prob $<F$ value and $F$-value analyzed by the analysis of variance (ANOVA), while the quality of the model was determined based on regression $\left(R^{2}\right)$, adjusted $R^{2}$, predicted $R^{2}$, lack of fit and adequate precision.

\subsection{Characterization}

The optimized immunosensor was characterized in a mixture solution of $5 \mathrm{mM} \mathrm{K}_{3}\left[\mathrm{Fe}(\mathrm{CN})_{6}\right], 5 \mathrm{mM} \mathrm{K}_{4}\left[\mathrm{Fe}(\mathrm{CN})_{6}\right]$, and $0.1 \mathrm{M} \mathrm{KCl}$ via $\mathrm{CV}(0.2 \mathrm{~V}$ to $0.6 \mathrm{~V}$ potential range) and electrochemical impedance spectroscopy (EIS) within the frequency range of $1 \mathrm{~Hz}$ to $10 \mathrm{MHz}$ using a sinusoidal current of $0.005 \mathrm{~V}$ amplitude at open circuit potential to evaluate its electrochemical behavior. Field emission scanning electron microscopy (FESEM, JEOL JSM-7600F) was performed to study its surface morphology. Confirmation of the $\mathrm{Ab}$ immobilization was determined by performing a bicinchoninic acid (BCA) protein assay. $^{49} 100 \mu \mathrm{L}$ of the substrate containing reagent $\mathrm{A}$ and reagent $B$ in a $9: 1$ ratio was added on each electrode surface for each fabrication stage. The electrodes were incubated for $30 \mathrm{~min}$ at $37{ }^{\circ} \mathrm{C}$ before the solutions were transferred to a 96well plate followed by absorbance reading at $560 \mathrm{~nm}$ using a microplate reader (VersaMax, Molecular Devices). The protein standard curve was obtained based on bovine serum albumin (BSA) protein. A stock solution of BSA $\left(5 \mathrm{mg} \mathrm{mL}^{-1}\right)$ was prepared in PBS and diluted to a series of standard solutions ( 0 to $4.0 \mathrm{mg}$ $\left.\mathrm{mL}^{-1}\right) .10 \mu \mathrm{L}$ per well of each solution was inserted into the 96well plate followed by $100 \mu \mathrm{L}$ of the substrate. The procedure was continued with incubation for $30 \mathrm{~min}$ at $37^{\circ} \mathrm{C}$ followed by absorbance reading at $560 \mathrm{~nm}$.

\subsection{Sample preparation for analysis of clenbuterol in meat samples}

Fresh beef samples were minced using a homogenizer and weighed $(0.1 \mathrm{~g})$ into centrifugal tubes. The samples were spiked with $1 \mathrm{~mL}$ of CLB standard (50 and $100 \mathrm{ng} \mathrm{mL}{ }^{-1}$ ) followed by the addition of $19 \mathrm{~mL}$ acetonitrile (ACN) and DI water. The samples were shaken using a shaker for $5 \mathrm{~min}$ before they were centrifuged at $3900 \mathrm{rpm}$ for $5 \mathrm{~min}$ at $4{ }^{\circ} \mathrm{C}$. The supernatants were transferred into centrifuge tubes containing $0.5 \mathrm{~g}$ LiChroprep ${ }^{\circledR} \mathrm{RP}-18$ (25-40 $\mu \mathrm{m}$, Merck), followed by 
the addition of $10 \mathrm{~mL}$ saturated hexane (saturated with ACN). The samples were shaken for another $1 \mathrm{~min}$ before centrifugation at $3900 \mathrm{rpm}$ for $5 \mathrm{~min}$ at $4{ }^{\circ} \mathrm{C}$. The upper layers containing hexane were removed. Only $5.0 \mathrm{~mL}$ of the aliquots was transferred into centrifuge tubes and evaporated to $1.0 \mathrm{~mL}$. The extracts were filtered with a 0.45 -micron nylon membrane syringe filter into vials for analysis via LCMS. Meanwhile, for analysis with the PEDOT/MWCNT/Ab/SPCE immunosensors, the extracts were evaporated until they were fully dried before dissolving them in buffer solution.

\section{Results and discussion}

\subsection{Immunosensing strategy}

In this study, SPCE underwent modification with PEDOT/ MWCNT before being further developed into an immunosensor (Fig. 1a). Our strategy was to directly electrodeposit the PEDOT/MWCNT composite instead of using the common covalent attachment of CNT to the electrode surface. ${ }^{50}$ To avoid entrapment of $\mathrm{Ab}$ into the polymer composite, ${ }^{51}$ only PEDOT and MWCNT were electrodeposited on the electrode. The Ab was covalently linked to the functionalized MWCNT in the next steps using the EDC/NHSS protocol. ${ }^{30}$ The MWCNT used for this modification contains abundant hydroxyl and carboxyl groups due to the functionalization with the acid mixture. The existence of carboxyl groups allows $\mathrm{Ab}$ to bind to the modified electrode surface through the EDC/NHSS crosslinker. The high surface area of MWCNT provide numerous binding sites for $\mathrm{Ab}$ to bind to the electrode surface, thus increasing the sensitivity of this immunosensor. Dry milk was used as a blocking agent to avoid unspecific binding on the PEDOT/MWCNT/Ab/SPCE surface. The blocking agent covered the electrode area that was not bound with $\mathrm{Ab}$ to avoid background noise from interrupting the current signal or giving any false signal due to unbound protein. Therefore, a washing step was included after each immobilization process to remove the unbound protein.

The CLB immunosensor developed in this study is based on the direct competitive detection method (Fig. 1b). Generally, CLB as a hapten has a very little effect on electron transfer. Label enzymes such as HRP are commonly used in immunoassay studies since their enzyme activity can be determined by fluorescence and electrochemical methods. To overcome the abovementioned problem, CLB conjugated with HRP (CLBHRP) was utilized to amplify the effect of electron transfer, which produces a measurable signal. In this study, the signal in the form of current value was measured based on the reduction of TMB catalyzed by HRP. The reduction of TMB is due to the catalyzation of CLB-HRP on the modified electrode surface resulting in a measurable electrochemical current signal. Sine CLB-HRP was competing with free CLB in the sample solution, the signal current produced was variable depending on the amount of CLB-HRP successfully bound to Ab on the modified electrode surface. As the concentration of free CLB in the sample increased, fewer CLB-HRP managed to bind with $\mathrm{Ab}$ immobilized on the electrode surface, and vice versa.

\subsection{Determination of set potential}

Since this study utilized CA to generate a signal for the detection of CLB, the optimum applied potential was investigated. The signal current over background current (S/B) ratio using step amperometry from -0.6 to $0.6 \mathrm{~V}$ for 0 to $100 \mathrm{ng} \mathrm{mL}^{-1} \mathrm{CLB}$ concentration was calculated and the graph was plotted (Fig. 1c) to observe the pattern of the current signal. Even though various concentrations of CLB produced different S/B ratios, the highest values were obtained at the same applied potential. These results show that $-0.1 \mathrm{~V}$ is the best potential for this system due to its largest $\mathrm{S} / \mathrm{B}$ ratio among the potentials measured at different CLB concentrations. Thus, $-0.1 \mathrm{~V}$ was chosen as the applied potential for further measurements.

\subsection{Optimization of the immunoassay conditions}

3.3.1 Optimization of Ab concentration. The sensitivity of immunosensors is highly dependent on the concentration of $\mathrm{Ab}$. In this study, the affinity between $\mathrm{Ab}$ and CLB was evaluated from indirect ELISA to determine the $\mathrm{Ab}$ titer. The Ab titer was determined based on the lowest concentration of $\mathrm{Ab}$ where the $\mathrm{Ab}-\mathrm{CLB}$ (the targeted antigen) reaction occurs. ${ }^{52}$ The titer value of $\mathrm{Ab}$ was determined as 1 : 10000 based on Fig. 1d, whereby this high $\mathrm{Ab}$ dilution indicates the high $\mathrm{Ab}$ activity towards the target (CLB).$^{53}$ Three concentrations of $\mathrm{Ab}(0.01,0.1$ and $1.0 \mathrm{mg}$ $\left.\mathrm{mL}^{-1}\right)$ were shortlisted based on the result of the ELISA titer (Fig. 1d). The standard CLB calibration curve for each $\mathrm{Ab}$ concentration was plotted (Fig. 1e) and the efficiency of the immunosensor was evaluated based on the $R^{2}$ value of the calibration curve. An ideal calibration curve has an $R^{2}$ value closer to 1.000. Based on Fig. 1e, the immunosensor developed using $0.1 \mathrm{mg} \mathrm{mL}{ }^{-1} \mathrm{Ab}$ has a better $R^{2}(0.9381)$ than $0.01 \mathrm{mg}$ $\mathrm{mL}^{-1} \mathrm{Ab}\left(R^{2}=0.8746\right)$. As the concentration of $\mathrm{Ab}$ increases, there are more chances for the formation of $\mathrm{Ab}-\mathrm{Ag}$ binding, thus increasing the immunosensor efficiency. However, when the concentration of $\mathrm{Ab}$ was increased to $1.0 \mathrm{mg} \mathrm{mL}^{-1}$, the $R^{2}$ significantly decreased to 0.3317 . This phenomenon occurred because saturation of the binding sites was achieved. A further increase in the $\mathrm{Ab}$ concentration to more than $0.1 \mathrm{mg} \mathrm{mL}$ does not increase the immunosensor efficiency, thus the $R^{2}$ does not improve. Consequently, the concentration of $0.1 \mathrm{mg}$ $\mathrm{mL}^{-1} \mathrm{Ab}$ was chosen as the optimum $\mathrm{Ab}$ concentration.

\subsection{Optimization of $\mathrm{pH}$, incubation temperature, $\mathrm{Ag}$ incubation time and \% blocking}

3.4.1 Model fitting and statistical analysis of result. The modelling and optimization of $\mathrm{pH}$, incubation time, $\mathrm{Ag}$ incubation time and blocking\% using RSM/CCD was performed to maximize the immunosensor current response in the detection of CLB followed by determination of the optimum electrochemical immunoassay conditions. The experimental data in Table $\mathrm{S} 1 \uparrow$ were analyzed and the significance of the model was determined based on the model $F$-value of 20.63 and value prob $>F$ of less than 0.0001, as shown in the ANOVA table (Table S $2 \dagger$ ). A prob $>F$ value less than 0.0500 indicates a significant model term, while a value greater than 0.1000 represents an 


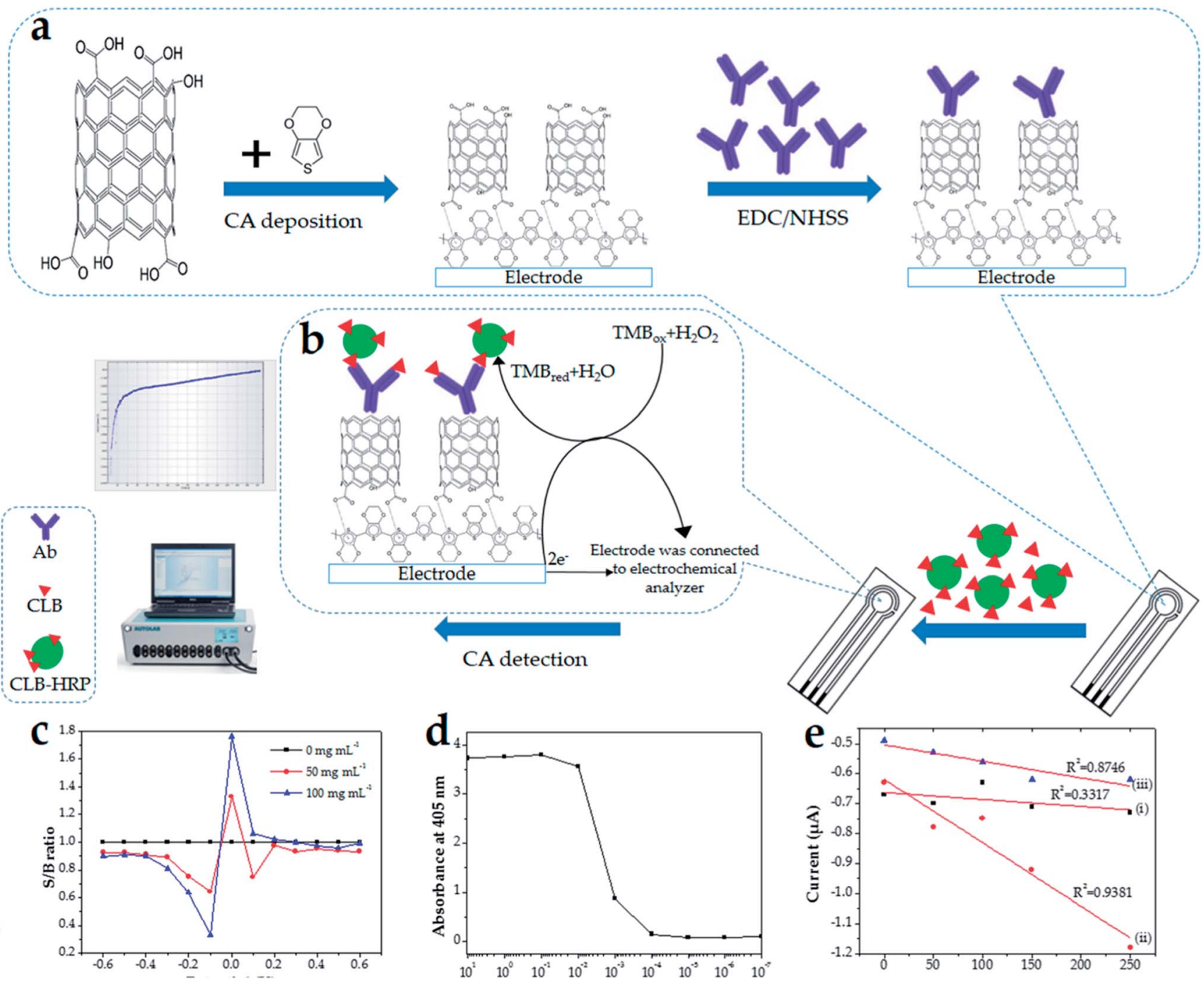

Fig. 1 (a) Schematic diagram of the fabrication of the CLB immunosensor. (b) Direct competitive detection method. (c) Plot signal to background $(\mathrm{S} / \mathrm{B})$ for each step potential from -0.6 to $0.6 \mathrm{~V}$ with chronoamperometry measurement (scan rate $=0.1 \mathrm{~V} \mathrm{~s}{ }^{-1}$ ) at various concentrations $(0,50$ and $100 \mathrm{ppb}$ ). (d) ELISA titer: Ab activity. (e) Standard CLB calibration graphs at different Ab concentrations of (i) $1.0 \mathrm{mg} \mathrm{mL}^{-1}$; (ii) $0.1 \mathrm{mg} \mathrm{mL}^{-1}$ and (iii) $0.01 \mathrm{mg} \mathrm{mL}^{-1}$ (scan rate $=0.1 \mathrm{~V} \mathrm{~s}^{-1}$ ).

insignificant model term. The analyzed data adequately fits the quadratic equation model with $R^{2}$, adjusted $R^{2}$ and predicted $R^{2}$ values of $0.9633,0.9166$ and 0.7573 , respectively, which fulfilled the acceptable requirement for at least 0.80 for $R^{2}$ and adjusted $R^{2} .{ }^{54}$ The values of standard deviation (SD) and prediction error sum of squares (PRESS) calculated were 0.030 and 0.066 , which are close to zero, also an indication for a good model. The experimental response was modeled as a polynomial equation, which represents the effect of experimental factors on the current response, as given in eqn (3):

Current $=0.146+0.002 A+0.052 B-0.052 C-0.019 D-0.037 A^{2}$ $+0.059 B^{2}-0.146 C^{2}-0.039 D^{2}+0.012 A B-0.020 A C$

$$
-0.004 A D-0.007 B C-0.003 B D+0.008 C D
$$

Four independent variables, i.e. $\mathrm{pH}$, incubation time, $\mathrm{Ag}$ incubation time and \% blocking, are represented by coded values ( $X_{1}=A, X_{2}=B, X_{3}=C$, and $X_{4}=D$, respectively). In this study, $B, C, D, B^{2}, C^{2}$ and $A C$ were indicated as significant model terms due to their prob $>F$ values of less than 0.0500 . Factor $\mathrm{pH}$ (model term A) was identified as an insignificant term, indicating the pH range of 6.40 to 8.40 does not affect the current response.

3.4.2 Effect of each factor individually and in interaction. The effect of the factors can be evaluated individually (Fig. 2) and/or by the interaction between factors through RSM. The influence of $\mathrm{pH}$ on this electrochemical immunoassay was investigated (Fig. 2a). Only a slight increase in current was determined as $\mathrm{pH}$ increased from 6.4 to 7.4 , followed by a slight current decrease from $\mathrm{pH} 7.4$ to 8.4. This graph shows that a $\mathrm{pH}$ value in range 6.4 to 8.4 is not significant. Based on ANOVA, the $F$-value for $\mathrm{pH}$ is low (0.10) and the prob $>F$ value is 0.7556 , which is greater than 0.1000 . Thus, the factor $\mathrm{pH}$ in the range from 6.4 to 8.4 is statistically not significant. 

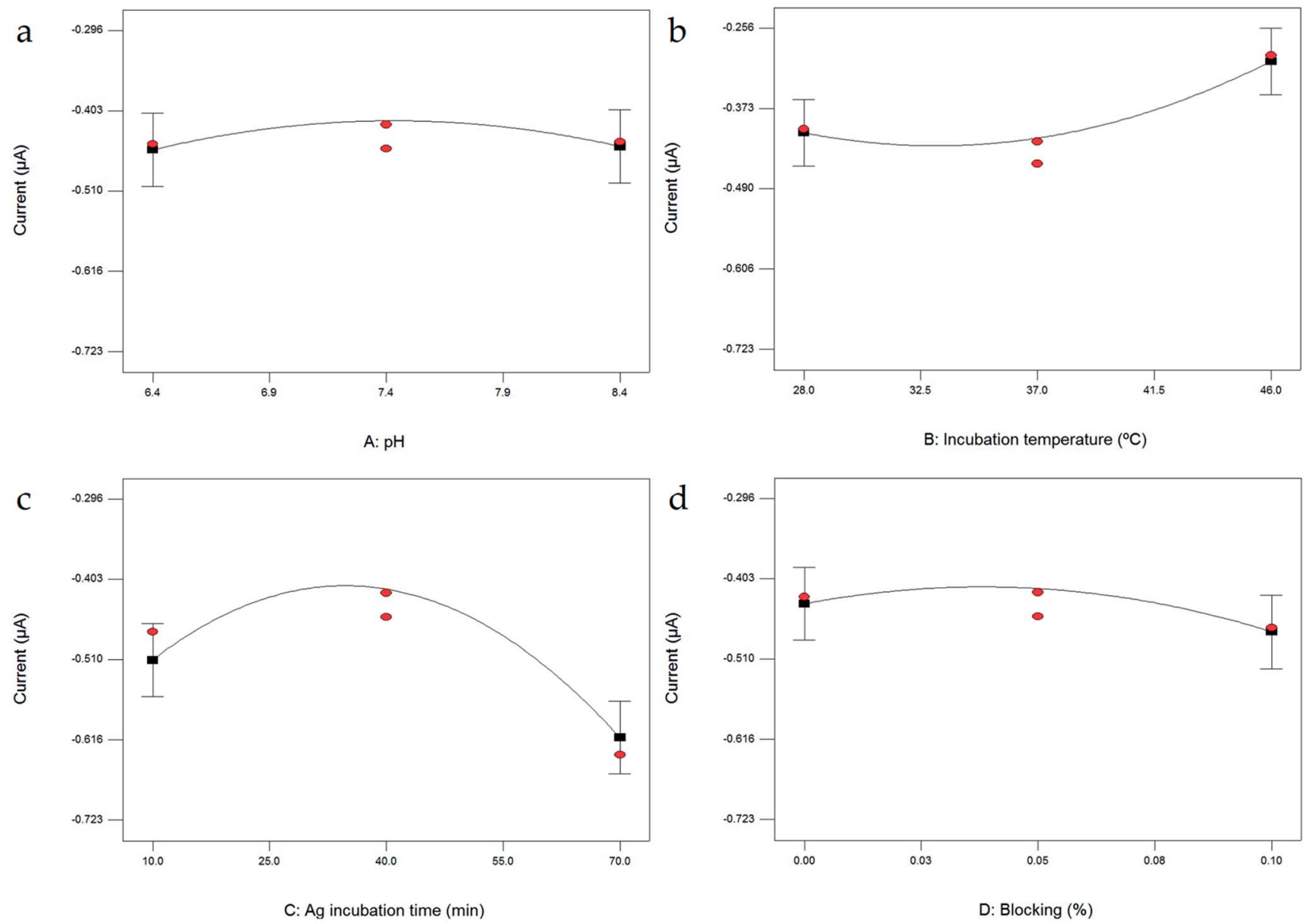

Fig. 2 One factor plots of peak current response as a function of (a) $\mathrm{pH}$; (b) incubation temperature; (c) Ag incubation time; and (d) blocking.

In this study, incubation temperature was evaluated from $28^{\circ} \mathrm{C}$ to $46^{\circ} \mathrm{C}$ (Fig. $2 \mathrm{~b}$ ). The reactivity of $\mathrm{Ab}$ and $\mathrm{Ag}$ is strongly affected by temperature. A very small current change occurred as the incubation temperature increased from $28^{\circ} \mathrm{C}$ to $37^{\circ} \mathrm{C}$, while the current obviously increased as the temperature increased to $46{ }^{\circ} \mathrm{C}$. The highest current reading observed at $46{ }^{\circ} \mathrm{C}$ shows that the $\mathrm{Ab}-\mathrm{Ag}$ interaction formed the best at this temperature. The formation of $\mathrm{Ab}-\mathrm{Ag}$ bonding also depends on the incubation time; thus, the incubation time of 10 to $70 \mathrm{~min}$. The current increases as the incubation time increases from 10

Table 1 Comparison between the original and optimized conditions for the electrochemical assay for the fabrication of the CLB immunoassay

\begin{tabular}{lll}
\hline & \multicolumn{2}{l}{$\begin{array}{l}\text { Fabrication of PEDOT/ } \\
\text { MWCNT/Ab/SPCE }\end{array}$} \\
\cline { 2 - 3 } Parameter & Original & Optimized \\
\hline $\mathrm{pH}$ & 7.4 & 7.78 \\
$\mathrm{Ab}$ incubation temperature $\left({ }^{\circ} \mathrm{C}\right)$ & 37 & 46 \\
Ag incubation time (min) & 60 & 33 \\
Blocking agent $(\%)$ & 0.05 & 0.03 \\
$R^{2}$ & 0.9381 & 0.9921
\end{tabular}

to $40 \mathrm{~min}$ (Fig. 2c), at which point, the highest current response was observed. However, the current reading decreases as the incubation time increases to $70 \mathrm{~min}$, showing that an incubation time longer than $40 \mathrm{~min}$ is not effective.

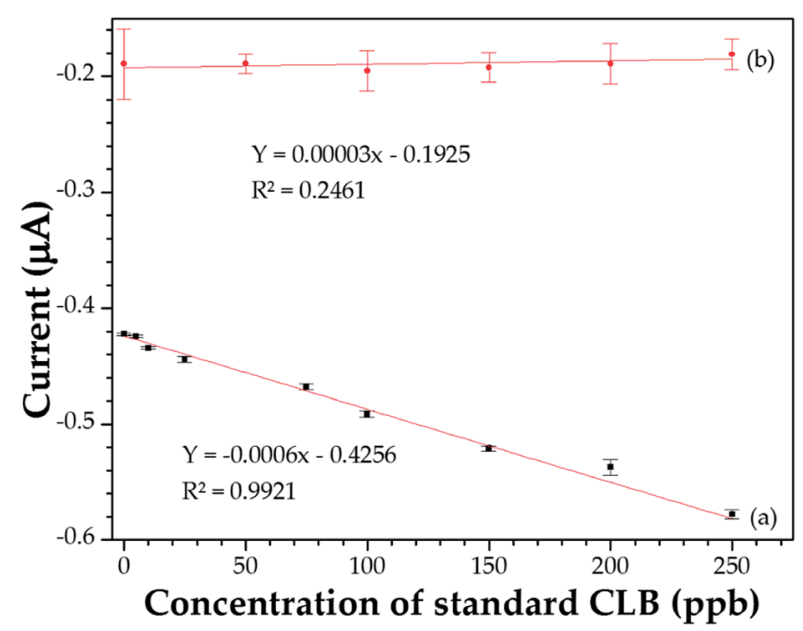

Fig. 3 Standard CLB calibration curve after optimization with RSM. (a) Immunosensor modified with PEDOT/MWCNT. (b) Sensor without MWCNT. 
Table 2 Comparison of the performances of the developed immunosensor for CLB detection with previous reports ${ }^{a}$

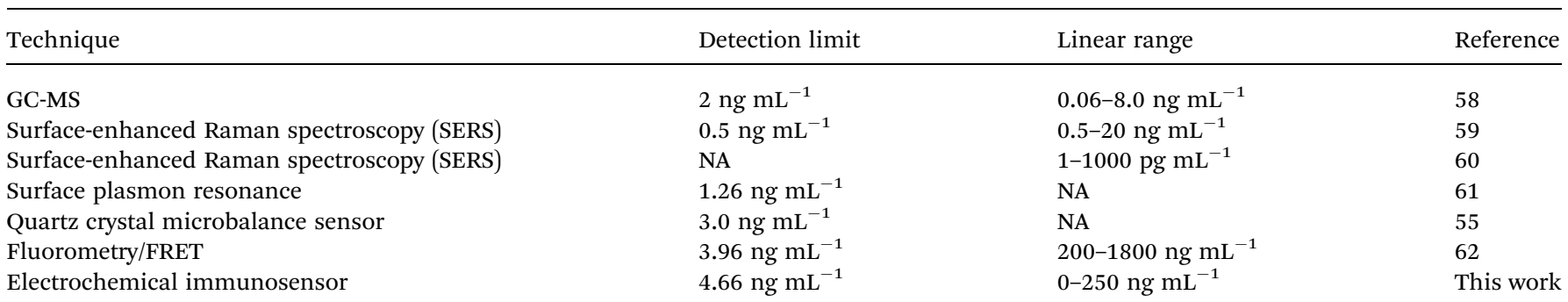

${ }^{a}$ NA: not available.

To avoid unspecific binding of any unwanted molecules, dry milk was introduced as a blocking agent. The percentage of blocking was evaluated in the range of $0 \%$ to $0.10 \%$. An increase in current was observed as the blocking was increased to $0.05 \%$ (Fig. 2d). As the blocking was further increased to $0.10 \%$, the current response decreased. An increase in blocking agent of more than $0.05 \%$ not only blocked the empty electrode surface but also caused current interruption. Thus, blocking agent of more than $0.05 \%$ is not necessary.

One of the advantages of RSM is its specialty to allow the interaction between factors to be studied. The interaction between factors is presented in Fig. S1.† Individually, the evaluation of $\mathrm{pH}$ shows no significant terms based on the graph (Fig. 2a) and statistical analysis (Table S2 $\dagger$ ). However, based on ANOVA (Table S2 $\dagger$ ), as the interaction between factors was evaluated in the term AC (representing the interaction between $\mathrm{pH}$ and $\mathrm{Ag}$ incubation time), the prob $>F$ value is 0.0233 . The $\mathrm{pH}$ value was affecting the $\mathrm{Ag}$ incubation time significantly. Thus, the study of the $\mathrm{pH}$ factor cannot be ignored even though individually this factor is considered as not significant.

3.4.3 Validation of model and optimization of current. Determination of the optimum conditions in the production of the highest current signal was performed by utilizing the Design Expert 6.0 software. The desired conditions were set with all the factors considered in the study range, while the response was set to the maximum current response. The optimum conditions predicted were $\mathrm{pH} 7.78,46^{\circ} \mathrm{C}$ for $\mathrm{Ab}$ incubation temperature, $33 \mathrm{~min} \mathrm{Ag}$ incubation time and $0.03 \%$ blocking agent. A set of experiments was performed to produce a calibration curve for the CLB standard using the predicted optimum conditions. This predicted optimum condition suggests a shorter $\mathrm{Ab}$ incubation time and a lower percentage of blocking agent in comparison with the original conditions (Table 1). The graph of CLB standard obtained under the optimized electrochemical immunoassay experimental conditions (Fig. 3a) reveals an improved $R^{2}$ value (0.9921). The LOD reported in this study was $4.66 \mathrm{ng} \mathrm{mL}{ }^{-1}$, which is lower than the Codex Alimentarius Commission regulations residue limit $\left(10 \mathrm{ng} \mathrm{mL}^{-1}\right) .{ }^{55}$ Meanwhile the calibration plot for the sensor without MWCNT (Fig. 3b) shows no significant current change at the studied CLB concentration range ( 0 to $250 \mathrm{ppb}$ ) with a near zero current reading, which indicates its very poor electrochemical sensing performance in comparison to the modified electrode as a result of no significant absorption of $\mathrm{Ab}$ on the unmodified
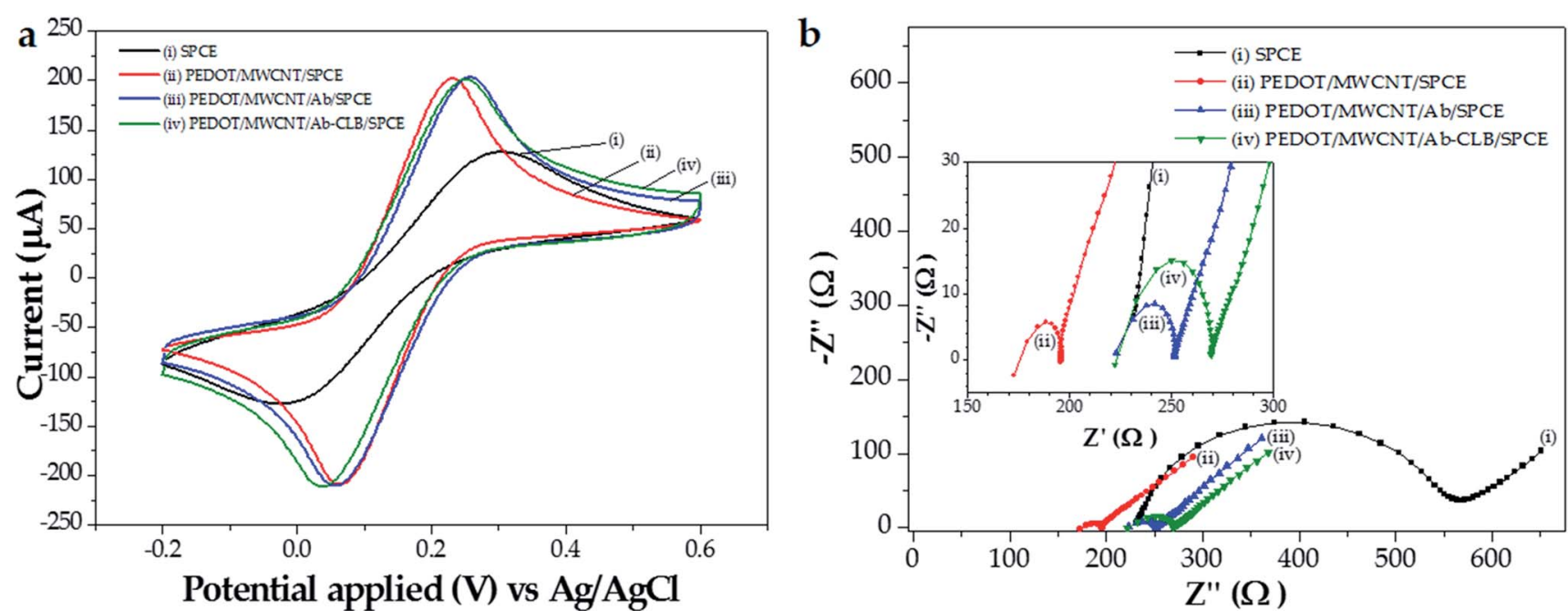

Fig. 4 (a) CV of (i) bare SPCE, (ii) PEDOT/MWCNT/SPCE, (iii) PEDOT/MWCNT/Ab/SPCE and (iv) PEDOT/MWCNT/Ab-CLB/SPCE in a mixture solution of $5 \mathrm{mM} \mathrm{K}_{3}\left[\mathrm{Fe}(\mathrm{CN})_{6}\right], 5 \mathrm{mM} \mathrm{K}_{4}\left[\mathrm{Fe}(\mathrm{CN})_{6}\right]$, and $0.1 \mathrm{M} \mathrm{KCl}$ (scan rate $=0.1 \mathrm{~V} \mathrm{~s}^{-1}$ ). (b) Nyquist plots of (i) bare SPCE, (ii) PEDOT/MWCNT/SPCE, (iii) PEDOT/MWCNT/Ab/SPCE, and (iv) PEDOT/MWCNT/Ab-CLB/SPCE in a mixture solution of $5 \mathrm{mM} \mathrm{K}_{3}\left[\mathrm{Fe}(\mathrm{CN})_{6}\right], 5 \mathrm{mM} \mathrm{K}{ }_{4}\left[\mathrm{Fe}(\mathrm{CN})_{6}\right]$, and $0.1 \mathrm{M}$ $\mathrm{KCl}$ (frequency range $=10 \mathrm{MHz}$ to $1 \mathrm{~Hz}$; amplitude $=0.005 \mathrm{~V}$ ); inset: magnified view of the high frequency region. 

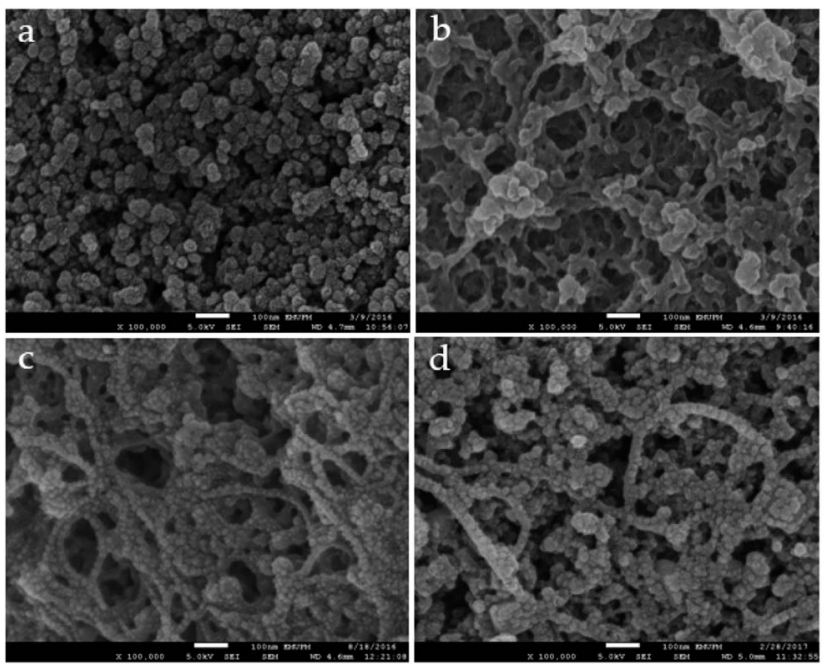

Fig. 5 FESEM images of (a) bare SPCE, (b) PEDOT/MWCNT/SPCE, (c) PEDOT/MWCNT/Ab/SPCE and (d) PEDOT/MWCNT/Ab-CLB/SPCE; magnification $100000 \times$.

electrode. LOD is defined as the concentration of CLB corresponding to the signal intensity, which is equal to the mean signal intensity of zero concentration minus three times the SD. ${ }^{56}$ The developed immunosensor in this study was compared with other CLB detection methods reported in the literature (Table 2), and it can be seen that the results obtained from our developed immunosensor is comparable with other studies.

\subsection{Characterization}

3.5.1 Cyclic voltammetry. The electrochemical behavior of the electrodes at each fabrication stage was studied via cyclic voltammetry (Fig. 4a). After the modification of SPCE with PEDOT/MWCNT, an increase in peak current was observed $\left(I_{\mathrm{pa}}\right.$ $=208 \mu \mathrm{A}$ and $\left.I_{\mathrm{pc}}=196 \mu \mathrm{A}\right)$, indicating the synergistic effect of the conducting polymer PEDOT and MWCNT successfully increased the conductivity of the electrode, thus resulting in an improved sensor platform. ${ }^{13,38}$ However, the peak current slightly decreased $\left(I_{\mathrm{pa}}=193 \mu \mathrm{A}\right.$ and $\left.I_{\mathrm{pc}}=-185 \mu \mathrm{A}\right)$ as Ab was immobilized onto the electrode surface and further decreased when CLB bound to the immobilized $\mathrm{Ab}\left(I_{\mathrm{pa}}=190 \mu \mathrm{A}\right.$ and $I_{\mathrm{pc}}=$ $-176 \mu \mathrm{A})$. Both $\mathrm{Ab}$ and CLB are non-conducting materials and thus insulate the modified electrode surface, which leads to a decrease in peak current as a result of electrode charge transfer interruption. ${ }^{57}$

3.5.2 Electrochemical impedance spectroscopy. The interfacial properties of the electrodes were further evaluated via EIS experiments. The impedance spectra (Fig. 4b) show linear a portion in the low frequency region and a semicircle portion in the high frequency region. The semicircle portion represents
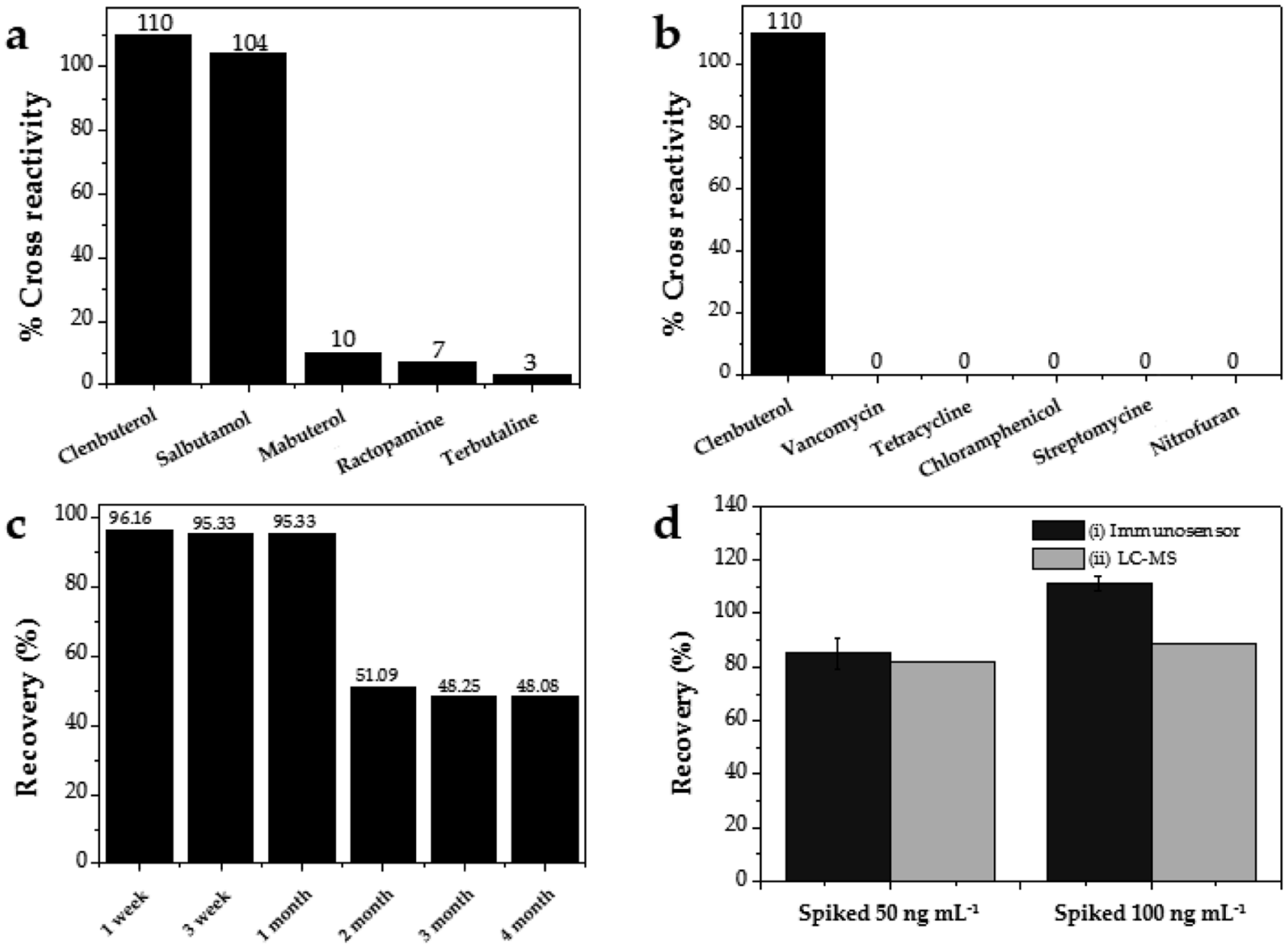

Fig. 6 Immunosensor selectivity against other antibiotics from (a) $\beta$-agonist family and (b) other groups. (c) Storage stability at $4{ }^{\circ} \mathrm{C}$. (d) Percentage recovery of CLB (i) immunosensor and (ii) LC-MS. 
Table 3 Antibiotic structures

Family Antibiotic $\quad$ Structure

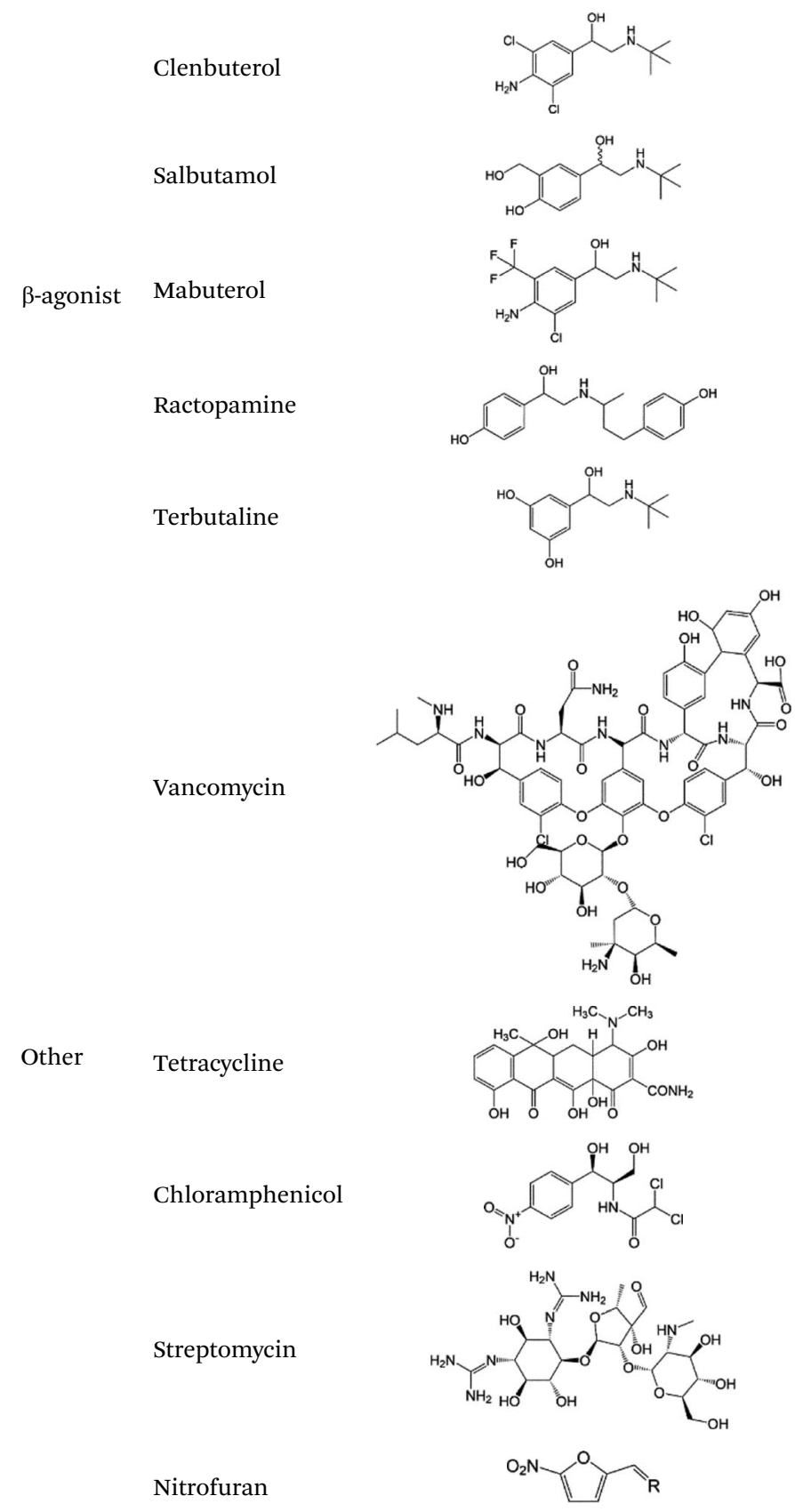

the charge transfer resistance $\left(R_{\mathrm{ct}}\right)$ according to its diameter. The bare SPCE displayed a large semicircle with an $R_{\mathrm{ct}}$ of $321 \Omega$. After the modification, a small semicircle was observed with an $R_{\text {ct }}$ of $20 \Omega$, implying an improvement in electrical conductivity and acceleration of the electron transfer rate of PEDOT/ MWCNT/SPCE. However, the $R_{\text {ct }}$ for PEDOT/MWCNT/Ab/SPCE increased to $32 \Omega$ due to the $\mathrm{Ab}$ immobilization on the modified electrode, which increases the impedance due to the formation of an additional barrier by $\mathrm{Ab}$ and prevents the electron transfer from the redox probe to the electrode surface. ${ }^{63}$
An enlargement in the semicircle was observed when CLB bound to $\mathrm{Ab}$, resulting in an $R_{\mathrm{ct}}$ of $44 \Omega$, indicating even higher transfer resistance occurred due to the existence of more nonfaradaic compounds on the electrode surface. EIS can be used to determine the existence of non-faradaic elements including $\mathrm{Ab}$ and CLB (analyte) on the electrode surface. ${ }^{64}$ The results show that the PEDOT/MWCNT composite is an excellent material, which improved the electrochemical performance of the modified electrode.

3.5.3 Morphology. The surface morphology after each modification stage was analyzed using FESEM at 100k magnification. Initially, SPCE had a rough surface, as shown in Fig. 5a. The treated MWCNT contained abundant carboxyl groups, thus allowing the negatively charged MWCNT to act as a dopant, which embedded into the polymer composite to balance the positive charge of the PEDOT backbone. Modification with PEDOT/MWCNT caused the SPCE surface to turn into a highly porous surface (Fig. 5b) due to the tubular structure of the MWCNT bound into the granular structure of PEDOT as a result of MWCNT doping into PEDOT. Even though the MWCNT doped into PEDOT through the carboxyl groups, there were still abundant unoccupied carboxyl groups that were exposed, thus allowing $\mathrm{Ab}$ to immobilize onto the modified electrode through the EDC/NHSS protocol. When Ab was immobilized onto the electrode surface, small granular structures formed on the smooth tubular structure of MWCNT, as shown in Fig. 5c, suggesting that these structures represent $\mathrm{Ab}$. Usually, protein such as $\mathrm{Ab}$ is not visible due to its transparent properties. However, as the samples were coated with platinum before the viewing process, thus $\mathrm{Ab}$ became visible. The existence of $\mathrm{Ab}$ on the electrode surface was further confirmed with the BCA protein assay. After incubation with reagents $\mathrm{A}$ and $\mathrm{B}$, the green color of the mixed reagent on the bare SPCE and PEDOT/ MWCNT/SPCE surfaces remained unchanged because no protein was present. However, for PEDOT/MWCNT/Ab/SPCE and PEDOT/MWCNT/Ab-CLB/SPCE, the green colour of the mixed reagent turned into purple as a result of the reduction of copper $\left(\mathrm{Cu}^{2+}\right)$ to cuprous $\left(\mathrm{Cu}^{+}\right)$by $\mathrm{Ab}$ (protein) followed by the formation of a BCA/copper complex due to $\mathrm{Cu}^{+}$chelation with BCA. The immobilization of $\mathrm{Ab}$ on the electrode surface was confirmed by this assay. The morphology of PEDOT/MWCNT/ Ab-CLB/SPCE (Fig. 5d) is similar to that of PEDOT/MWCNT/Ab/ SPCE because the small CLB structure is not visible.

\subsection{Analytical performance of the immunosensor}

The specificity of this CLB immunosensor was evaluated by detected other antibiotics from the $\beta$-agonist group, such as salbutamol, mabuterol, ractopamine and terbutaline, along with other antibiotics from other groups such as vancomycin, tetracycline, chloramphenicol, streptomycin and nitrofuran. The current response of each antibiotic with the same concentration of $100 \mathrm{ng} \mathrm{mL} \mathrm{m}^{-1}$ was investigated. The inhibition percentages were calculated as $110 \%, 104 \%, 10 \%, 7 \%$ and $3 \%$ for CLB, salbutamol, mabuterol, ractopamine and terbutaline, respectively $(n=3)$ (Fig. 6a). 
Other antibiotics from other families such as vancomycin, tetracycline, chloramphenicol, streptomycin and nitrofuran $(n$ $=3$ ) show $0 \%$ cross reactivity (Fig. $6 \mathrm{~b}$ ). Other $\beta$-agonists and antibiotics do not affect the CLB detection except salbutamol, which implies that this immunosensor has good selectivity towards CLB. Salbutamol shows a high\% cross reactivity because its structure is closer to CLB than other antibiotics (Table 3).

The reproducibility of this immunosensor was determined for $n=10$ under the same conditions, resulting in the standard deviation (SD) value of 0.110 , indicating excellent reproducibility. The coefficient of variation of this reproducibility test is $1.33 \%$. The coefficient of variation is defined as the ratio of the standard deviation to mean [(SD/mean)100\%], where a value a less than $5 \%$, as reported in this study, implies excellent reproducibility. The immunosensor was also stored at $4{ }^{\circ} \mathrm{C}$ for one month to study its storage stability. After 1 month of storage, $95.33 \%$ (Fig. 6c) of the initial current response was obtained, indicating relatively good storage stability.

\subsection{Comparative study}

Real sample analyses were performed to detect CLB in 10 beef samples using the developed CLB immunosensor. The same samples were also detected using LC-MS for comparison. Since no CLB was detected, standard solutions of CLB were spiked in the samples to evaluate the applicability of the developed immunosensor. As shown in Fig. 6d, good recoveries with 85\% to $111 \%$ recovery obtained for both the 50 and $100 \mathrm{ng} \mathrm{mL}$ spiked samples, respectively, as detected by the immunosensor. Comparable results were obtained from this immunosensor with that from LC-MS, which proves the reliability of this immunosensor for CLB detection in real samples.

\section{Conclusions}

A PEDOT/MWCNT/Ab/SPCE immunosensor was successfully developed for the detection of CLB. The optimum conditions for the electrochemical immunoassay were determined using RSM/ CCD. Incubation time, Ag incubation time and \% blocking were determined as significant parameters along with the interaction between $\mathrm{pH}$ and $\mathrm{Ag}$ incubation time. A linear graph of CLB standard with $R^{2}=0.9921$ was obtained under the optimized electrochemical immunoassay conditions. Modification of SPCE with the PEDOT/MWCNT composite improved the electrochemical properties of the electrode and provided a high surface area for Ab-CLB binding. Consequently, high reproducibility, storage stability and selectivity towards CLB was produced. This method was successfully employed for CLB detection in real meat samples, which produced comparable results with LC-MS, and thus is reliable for CLB screening and monitoring in real applications.

\section{Conflicts of interest}

There are no conflicts to declare.

\section{Acknowledgements}

The authors would like to thank Fundamental Research Grant Scheme (01-01-15-1707FR) and Malaysian Agricultural Research and Development Institute (MARDI) for funding this research project.

\section{References}

1 L. A. Cudmore, T. Muurlink, T. Whittem and S. R. Bailey, Res. Vet. Sci., 2013, 94, 682-686.

2 D. S. Huckins and M. F. Lemons, J. Emerg. Med, 2013, 44, 444-449.

3 C. T. Elliott, S. R. H. Crooks, J. G. D. McEvoy, W. J. McCaughey, S. A. Hewitt, D. Patterson and D. Kilpatrick, Vet. Res. Commun., 1993, 17, 459-468.

4 G. Brambilla, T. Cenci, F. Franconi, R. Galarini, A. Macrì, F. Rondoni, M. Strozzi and A. Loizzo, Toxicol. Lett., 2000, 114, 47-53.

5 A. Posyniak, J. Zmudzki and J. Niedzielska, Anal. Chim. Acta, 2003, 483, 61-67.

6 B. Liu, H. Yan, F. Qiao and Y. Geng, J. Chromatogr. B: Anal. Technol. Biomed. Life Sci., 2011, 879, 90-94.

7 K. Yan, H. Zhang, W. Hui, H. Zhu, X. Li, F. Zhong, X. e. Tong and C. Chen, J. Food Drug Anal., 2016, 24, 277-283.

8 R. Nicoli, M. Petrou, F. Badoud, J. Dvorak, M. Saugy and N. Baume, J. Chromatogr. A, 2013, 1292, 142-150.

9 L. Amendola, C. Colamonici, F. Rossi and F. Botrè, J. Chromatogr. B: Anal. Technol. Biomed. Life Sci., 2002, 773, 716.

10 J. Zhou, X. Xu and Y. Wang, J. Chromatogr. B: Anal. Technol. Biomed. Life Sci., 2007, 848, 226-231.

11 H. Wang, H. Yan, M. Shi, F. Wang, K. Mei, Q. Yu, X. Lv and X. Wang, Anim. Feed Sci. Technol., 2015, 209, 257-267.

12 S. Suherman, K. Morita and T. Kawaguchi, Appl. Surf. Sci., 2015, 332, 229-236.

13 P. Zhan, X.-W. Du, N. Gan, S.-C. Lin, T.-H. Li, Y.-T. Cao and W.-G. Sang, Anim. Feed Sci. Technol., 2013, 41, 828-834.

14 M. Sheikhshoaie, I. Sheikhshoaie and M. Ranjbar, J. Mol. Liq., 2017, 231, 597-601.

15 N. K. Bhajanthri, V. K. Arumugam, R. Chokkareddy and G. G. Redhi, J. Mol. Liq., 2016, 222, 370-376.

16 X.-P. Wei, Y.-L. Luo, F. Xu, Y.-S. Chen and L.-H. Yang, Mater. Des., 2016, 111, 445-452.

17 P. B. Deroco, B. C. Lourencao and O. Fatibello-Filho, Microchem. J., 2017, 133, 188-194.

18 P. Devi, G. Kaur, A. Thakur, N. Kaur, A. Grewal and P. Kumar, Talanta, 2017, 170, 49-55.

19 A. Ferancová, M. K. Hattuniemi, A. M. Sesay, J. P. Räty and V. T. Virtanen, J. Hazard. Mater., 2016, 306, 50-57.

20 Y. Zhou, L. Yang, S. Li and Y. Dang, Sens. Actuators, B, 2017, 245, 238-246.

21 R. Sha, K. Komori and S. Badhulika, Electrochim. Acta, 2017, 233, 44-51.

22 M. Roushani, A. Nezhadali, Z. Jalilian and A. Azadbakht, Mater. Sci. Eng., C, 2017, 71, 1106-1114. 
23 Y. Liu, L. Zhu, Y. Hu, X. Peng and J. Du, Food Chem., 2017, 221, 1128-1134.

24 A. J. Zaitouna and R. Y. Lai, Anal. Chim. Acta, 2014, 828, 8591.

25 S. Campuzano, P. Yánez-Sedeño and J. M. Pingarrón, Electrochim. Acta, 2017, 230, 271-278.

26 J. Zhou, X. Qiu, K. Su, G. Xu and P. Wang, Sens. Actuators, B, 2016, 235, 170-178.

27 D. Antuña-Jiménez, M. C. Blanco-López, A. J. MirandaOrdieres and M. J. Lobo-Castañón, Sens. Actuators, B, 2015, 220, 688-694.

28 F. Ricci, G. Volpe, L. Micheli and G. Palleschi, Anal. Chim. Acta, 2007, 605, 111-129.

29 W. Duan, G. Chen, C. Chen, R. Sanghvi, A. Iddya, S. Walker, H. Liu, A. Ronen and D. Jassby, J. Membr. Sci., 2017, 531, 160-171.

30 X. Yu, B. Munge, V. Patel, G. Jensen, A. Bhirde, J. D. Gong, S. N. Kim, J. Gillespie, J. S. Gutkind, F. Papadimitrakopoulos and J. F. Rusling, J. Am. Chem. Soc., 2006, 128, 11199-11205.

31 A. Juzgado, A. Soldà, A. Ostric, A. Criado, G. Valenti, S. Rapino, G. Conti, G. Fracasso, F. Paolucci and M. Prato, J. Mater. Chem. B, 2017, 5, 6681-6687.

32 M. Eising, C. E. Cava, R. V. Salvatierra, A. J. G. Zarbin and L. S. Roman, Sens. Actuators, B, 2017, 245, 25-33.

33 Y. Li, D. Ye, W. Liu, B. Shi, R. Guo, H. Pei and J. Xie, J. Colloid Interface Sci., 2017, 493, 241-248.

34 G. Xu, B. Li, X. T. Cui, L. Ling and X. Luo, Sens. Actuators, B, 2013, 188, 405-410.

35 L. Wilson, J. Vam Wyk, C. Rassie, N. Ross, C. Sunday, H. R. Makelane, M. Bilibana, T. Waryo, S. Mapolie, P. G. Baker and E. I. Iwuoha, Int. J. Electrochem. Sci., 2015, 10, 3207-3222.

36 A. Kros, S. W. F. M. van Hövell, N. A. J. M. Sommerdijk and R. J. M. Nolte, Adv. Mater., 2001, 13, 1555-1557.

37 K. Liu, J. Zhang, Q. Liu and H. Huang, Electrochim. Acta, 2013, 114, 448-454.

38 N. A. A. Talib, F. Salam, N. A. Yusof, A. S. Alang Ahmad and Y. Sulaiman, RSC Adv., 2017, 7, 11101-11110.

39 L. Wang, R. Yang, J. Chen, J. Li, L. Qu and P. d. B. Harrington, Food Chem., 2014, 164, 113-118.

40 F. Lopes, J. G. Pacheco, P. Rebelo and C. Delerue-Matos, Sens. Actuators, B, 2017, 243, 745-752.

41 Z. Taleat, A. Khoshroo and M. Mazloum-Ardakani, Microchim. Acta, 2014, 181, 865-891.

42 L. Mirmoghtadaie, A. A. Ensafi, M. Kadivar and P. Norouzi, Mater. Sci. Eng., C, 2013, 33, 1753-1758.
43 J. I. A. Rashid, N. A. Yusof, J. Abdullah, U. Hashim and R. Hajian, J. Mater. Sci., 2015, 51, 1083-1097.

44 Z. Urkut, P. Kara, Y. Goksungur and M. Ozsoz, Electroanalysis, 2011, 23, 2668-2676.

45 M. D. Gouda, M. S. Thakur and N. G. Karanth, World J. Microbiol. Biotechnol., 2001, 17, 595-600.

46 A. Khoobi, S. M. Ghoreishi, M. Behpour and S. Masoum, Anal. Chem., 2014, 86, 8967-8973.

47 Y. Dou, Z. Jiang, W. Deng, J. Su, S. Chen, H. Song, A. Aldalbahi, X. Zuo, S. Song, J. Shi and C. Fan, J. Electroanal. Chem., 2016, 781, 339-344.

48 Y. Lai, J. Bai, X. Shi, Y. Zeng, Y. Xian, J. Hou and L. Jin, Talanta, 2013, 107, 176-182.

49 J. M. Walker, in The Protein Protocols Handbook, ed. J. M. Walker, Humana Press, Totowa, NJ, 2002, vol. 11, pp. 1114, DOI: $10.1385 / 1-59259-169-8$.

50 F. Patolsky, Y. Weizmann and I. Willner, Angew. Chem., Int. Ed., 2004, 43, 2113-2117.

51 S. Cosnier and M. Holzinger, Chem. Soc. Rev., 2011, 40, 2146. 52 B. H. Shaz, in Transfusion Medicine and Hemostasis, Elsevier, San Diego, 2nd edn, 2013, pp. 307-315, DOI: 10.1016/B978-012-397164-7.00048-3.

53 M. Johnson, Anal. Chem., 2012, 2, 156.

54 T. Lundstedt, E. Seifert, L. Abramo, B. Thelin, Å. Nyström, J. Pettersen and R. Bergman, Chemom. Intell. Lab. Syst, 1998, 42, 3-40.

55 F. Feng, J. Zheng, P. Qin, T. Han and D. Zhao, Talanta, 2017, 167, 94-102.

56 J. Kong, L. Jiang, X. Su, J. Qin, Y. Du and B. Lin, Lab Chip, 2009, 9, 1541.

57 X. Yang, F. Wu, D.-Z. Chen and H.-W. Lin, Sens. Actuators, B, 2014, 192, 529-535.

58 S. Yang, X. Liu, Y. Xing, D. Zhang, S. Wang, X. Wang, Y. Xu, M. Wu, Z. He and J. Zhao, J. Chromatogr. Sci., 2012, 00, 1-10.

59 J. Cheng, X.-O. Su, S. Wang and Y. Zhao, Sci. Rep., 2016, 6, 32637.

60 M. Yu, Y. Hu and J. Liu, New J. Chem., 2017, 41, 10407-10414.

61 J. Hu, R. Chen, S. Wang, T. Wang, Y. Zhao, J. Li, X. Hu, H. Liang, J. Zhu, X. Sun, L. Ma and M. Jiang, PLoS One, 2015, 10, e0122005.

62 J. Xu, Y. Li, J. Guo, F. Shen, Y. Luo and C. Sun, Food Control, 2014, 46, 67-74.

63 P. Yan, Q. Tang, A. Deng and J. Li, Sens. Actuators, B, 2014, 191, 508-515.

64 V. Nandakumar, J. T. La Belle, J. Reed, M. Shah, D. Cochran, L. Joshi and T. L. Alford, Biosens. Bioelectron., 2008, 24, 10451048. 